\section{Anestesiología}

Enero-Marzo 2022

Vol. 45. No. 1. pp 30-34

doi: $10.35366 / 102900$

\title{
Efecto de la maniobra de fijación en línea en la clasificación de Cormack-Lehane
}

\section{Effect of the in-line fixation maneuver on Cormack-Lehane classification}

\author{
Dra. Mercedes Nicté López-Hernández,* Dr. Eduardo A Wilson-Manríquez, \\ Dr. Francisco Alejandro López-Jiménez ${ }^{\S}$
}

Citar como: López-Hernández MN, Wilson-Manríquez EA, López-Jiménez FA. Efecto de la maniobra de fijación en línea en la clasificación de Cormack-Lehane. Rev Mex Anestesiol. 2022; 45 (1): 30-34. https://dx.doi. org/10.35366/102900

\begin{abstract}
RESUMEN. Introducción: El manejo correcto de la vía aérea en los pacientes politraumatizados es crucial, ya que es necesario tener una vía aérea segura y proveer adecuada ventilación sin emperorar una probable lesión medular. Objetivo: Determinar el efecto de la maniobra de fijación en línea (MILS del inglés Manual In-Line Stabilisation) en la clasificación de Cormack-Lehane (CL), así como la correlación con el índice de masa corporal (IMC). Material y métodos: En un estudio descriptivo en el Centro Hospitalario del Estado Mayor Presidencial en la Ciudad de México se incluyeron 56 pacientes con estado físico ASA I a IV. El anestesiólogo realizó la laringoscopía directa bajo MILS y valoró el grado de CL. Inmediatamente después se reposicionó al paciente en posición de olfateo, se efectuó nueva laringoscopía directa y se revaloró de nuevo el grado de CL. Resultados: Los grados del CL fueron significativamente diferentes entre la posición MILS versus olfateo. Los grados de CL fueron en su mayoría altos cuando se posicionó al paciente en MILS (75\% de los pacientes clasificados entre III y IV) y disminuyeron significativamente al ser cambiados a posición de olfateo. Conclusión: Se observa mejoría del CL cuando se cambia de posición MILS a olfateo.
\end{abstract}

\begin{abstract}
Introduction: Correct airway management of polytraumatized patients is crucial because of the necessity of securing the airway and providing adequate ventilation without worsening a probable spinal cord injury. Objective: Determine the effect of manual inline stabilization (MILS) on Cormack-Lehane classification and if there is any correlation with body mass index (BMI). Material and methods: In a descriptive study at the Centro Hospitalario del Estado Mayor Presidencial in Mexico City, we included 56 patients with ASA physical status I to IV. The anesthesiologist performed direct laryngoscopy while MILS was performed and observed the CL grade. Immediately after, the patient was repositioned into the sniffing position, direct laryngoscopy was performed, and the CL grade was observed again. Results: The CL grades observed were significantly different between MILS vs. Sniffing position. CL grades were mainly high when positioned in MILS (75\% classified as grades III and IV) and diminished significantly when changed to the sniffing position. Conclusion: Improvement of CL grade was observed when changing from MILS to sniffing position.
\end{abstract}

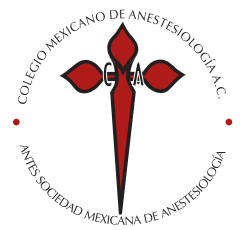

Palabras clave:

Traumatismo columna cervical, laringoscopía difícil, intubación difícil, videolaringocopio, maniobra fijación en línea, lesión medular.

Keywords:

Cervical spine trauma, difficult laryngoscopy, difficult intubation, videolaryngoscope, manual in-line stabilization, spinal cord injury.

* R3 Anestesiología. Hospital Ángeles Lomas, Huixquilucan, Estado de México, México.

‡ R2 Anestesiología, Instituto Nacional de Ciencias Médicas y Nutrición «Salvador Zubirán», Tlalpan, Ciudad de México, México. $\S$ Coronel Neuroanestesiólogo, Hospital Ángeles Lomas, Huixquilucan, Estado de México, México.

Correspondencia:

Dra. Mercedes Nicté

López-Hernández

Tel: 55 3567-4471

E-mail: wniclop.09@gmail.com

Recibido: 11-05-2021

Aceptado: 12-08-2021

\section{INTRODUCCIÓN}

A proximadamente 5.8 millones de personas alrededor del mundo fallecen anualmente a causa de lesiones traumáticas y violencia, lo que corresponde a $10 \%$ de las muertes en todo el mundo ${ }^{(1)}$. El paciente con trauma múltiple es un reto diagnóstico y terapéutico, 2-5\% de los pacientes con trauma contundente ${ }^{(2)}$ presentan lesiones de médula espinal cervical y puede ser aún más alto en pacientes con trauma severo. El manejo de la vía aérea en estos pacientes es crucial, ya que es necesario tener una vía aérea segura y proveer adecuada ventilación sin causar más daño al paciente agravando una lesión medular durante la manipulación de la vía aérea.

La maniobra de posicionamiento en olfateo provee la mejor visión glótica; sin embargo, causa un movimiento máximo de las vértebras cervicales que puede ser peligroso en un paciente con una lesión cervical sospechada o confirmada. La técnica más empleada actualmente para estabilizar la columna cervical mientras se establece una vía aérea definitiva en pacientes con trauma agudo es MILS. Se recomendó por 


\begin{tabular}{lccrr} 
& \multicolumn{3}{c}{ Tabla 1: Descriptivos generales de la población. } \\
& & Mediana & Mínimo & Máximo \\
\hline Edad (años) & $41.1 \pm 19.1$ & 38.5 & 14.0 & 85.0 \\
Talla $(m)$ & $1.6 \pm 0.1$ & 1.6 & 1.4 & 1.8 \\
Peso $(\mathrm{kg})$ & $69.3 \pm 10.7$ & 68.3 & 49.0 & 102.0 \\
Índice de masa corporal & $27.2 \pm 4.5$ & 27.2 & 19.6 & 39.3
\end{tabular}

primera vez en el Manual de Soporte Vital Avanzado de Trauma en $1984^{(3)}$ y en la última edición de este manual (2018) sigue siendo una recomendación clave en el manejo de vía aérea en estos pacientes. La maniobra de MILS la realiza el asistente del anestesiólogo durante el manejo de la vía aérea una vez que se retira el collarín rígido, tiene como objetivo mantener la cabeza y el cuello en posición neutra y evitar movimientos inadvertidos que puedan causar o empeorar una lesión medular preexistente. El asistente se coloca a un lado del anestesiólogo, sostiene la cabeza entre ambas manos, toma con los dedos los procesos mastoideos y ejerce presión con las palmas sobre el hueso occipital, buscando igualar y oponer las fuerzas generadas por el profesional que realiza la intubación. Se ha descrito un aumento en la dificultad de realizar intubación orotraqueal con la aplicación de MILS(4).

Este estudio buscó simular MILS en pacientes mexicanos sanos y determinar el efecto de esta maniobra con el CL así como la correlación con el IMC.

\section{MATERIAL Y MÉTODOS}

Se realizó un estudio descriptivo, el cual fue aprobado por el Comité de Ética Hospitalaria del Centro Hospitalario del Estado Mayor Presidencial en la Ciudad de México. Se recabó la firma de consentimiento informado por parte del paciente. Se incluyeron 56 pacientes con estado físico ASA I a IV programados para cirugía electiva. Se excluyeron pacientes con vía aérea difícil predicha durante la valoración preanestésica, incluyendo $\geq$ dos de los siguientes: Mallampati grado IV, cuello visiblemente corto, distancia interincisiva $<3 \mathrm{~cm}$, distancia tiromentioniana $<6.5 \mathrm{~cm}$, distancia esternomentoniana $<12$ cm o extensión atlantooccipital disminuida.

Se realizó preoxigenación con $\mathrm{O}_{2}$ a $100 \%$ durante cinco minutos y posteriormente se efectuó inducción anestésica con $3 \mu \mathrm{g} / \mathrm{kg}$ de fentanilo, $2 \mathrm{mg} / \mathrm{kg}$ de propofol y cisatracurio 100 $\mu \mathrm{g} / \mathrm{kg}$. Se dieron cuatro minutos de histéresis farmacológica mientras se realizó ventilación por presión positiva. A continuación el anestesiólogo encargado de la intubación efectuó la laringoscopía directa bajo la maniobra de MILS (realizada por otro anestesiólogo experimentado), con hoja Macintosh \#3 valoró el CL y posteriormente se reposicionó al paciente en posición de olfateo para revalorar el CL. Nunca se realizó la maniobra de BURP (del ingles Backward, Upward, Rigth Lateral Position). En cada una de las dos maniobras se calificó la visión glótica con la clasificación de CL: grado I = mayoría de glotis visible, grado II = sólo cartílagos aritenoides visibles, grado III = epiglotis visible, grado IV = imposibilidad de ver glotis y epiglotis.

Se determinaron medidas de tendencia central, porcentajes y frecuencias. Se agrupó a los pacientes utilizando la clasificación de CL y se valoró la correlación del IMC con la mejoría en el CL. Para las comparaciones entre los grupos se realziaron pruebas de $\chi^{2}$, prueba de comparación de proporciones (z test) y análisis de varianza (ANOVA) de una vía. Se empleó un intervalo de confianza (IC) de $95 \%$ y se consideraron estadísticamente significativos los valores de $\mathrm{p}$ $<0.05$. Los análisis estadísticos se realizaron en el software SAS 3.8 (SAS Institute Inc., NC, USA) y en Sigma Plot ver. 12.0 (Systat Software Inc).

\section{RESULTADOS}

La población estudiada consistió en $53.57 \%$ femeninos ( $\mathrm{n}=$ $30)$ y $46.43 \%$ masculinos $(n=26)$. El rango de edad de los pacientes fue de 14 a 85 años (41.1 \pm 19.1 años) y pesaron desde 49 hasta $102 \mathrm{~kg}(69.3 \pm 10.7 \mathrm{~kg})$ (Tabla 1). Las vistas glóticas obtenidas durante la laringoscopía directa en posición MILS y olfateo se muestran en la Tabla 2. Las proporciones de pacientes clasificados en las cuatro diferentes categorías fueron significativamente diferentes entre posición MILS versus olfateo. (Prueba de $\chi^{2} \mathrm{p} \leq 0.001$; Anexo 1). Las clasificaciones de CL fueron en su mayoría altas cuando se posicionó al paciente en MILS (75\% de los pacientes clasificados entre III y IV). En contraste, las vistas glóticas clasificadas entre

Tabla 2: Distribución de frecuencias de clasificaciones Cormarck y Lehane en posición de Manual In-Line Stabilisation y posición de olfateo.

\begin{tabular}{ccc} 
Clasificación & MILS, $\mathrm{n}(\%)$ & Olfateo, $\mathrm{n}(\%)$ \\
\hline I & $1(1.79)$ & $39(69.64)$ \\
II & $13(23.21)$ & $10(17.86)$ \\
III & $26(46.43)$ & $6(10.71)$ \\
IV & $16(28.57)$ & $1(1.79)$
\end{tabular}




\begin{tabular}{lcc} 
& $\begin{array}{c}\text { Tabla 3: Mejoría presentada por los pacientes } \\
\text { después de modificar la posición de MILS a olfateo, } \\
\\
\text { agrupados por la clasificación inicial y final. }\end{array}$ \\
\hline MILS (inicial) & Olfateo (modificación) & $n(\%)$ \\
\hline I $(\mathrm{N}=1)$ & I & $1(100.0)$ \\
II $(\mathrm{N}=13)$ & $\mathrm{I}$ & $12(92.3)$ \\
& II & $1(7.7)$ \\
III $(\mathrm{N}=26)$ & $\mathrm{I}$ & $20(76.9)$ \\
& II & $5(19.2)$ \\
IV $(\mathrm{N}=16)$ & III & $1(3.9)$ \\
& I & $6(37.5)$ \\
& II & $4(25.0)$ \\
& III & $5(31.3)$ \\
& IV & $1(6.2)$ \\
\hline
\end{tabular}

MLLS = Manual In-Line Stabilisation.

III y IV de esos mismos pacientes cuando fueron cambiados a posición de olfateo, sólo representaron $12.5 \%$ del total. (Prueba de $\chi^{2} \mathrm{p} \leq 0.001$; Aпехо 2).

Del total de 56 pacientes, al realizar la laringoscopia directa cambiando de MILS a olfateo, el CL se mantiene igual en cuatro pacientes (7.14\%), disminuyó un grado en 24 pacientes (42.86\%), dos grados en 22 pacientes (39.29\%) y tres grados en seis pacientes (10.71\%) (Tabla 3). De los 16 pacientes con grado IV de Cormack y Lehane con posición MILS, al cambiar a posición de olfateo se observó cambio a grado I en seis de estos pacientes, a grado II en cuatro pacientes, a grado III en cinco pacientes y se mantuvo el grado IV en un paciente.

La media del IMC no fue significativamente diferente entre los grupos de CL en posición MILS y CL en posición de olfateo cuando se compararon mediante ANOVA de una vía (p $=0.1$; Anехо 3). No se encontraron diferencias significativas entre los valores de IMC de las diferentes combinaciones de mejoría al cambiar de posición MILS a olfateo al comparar mediante ANOVA de una vía $(\mathrm{p}=0.086$; Anexo 3).

\section{DISCUSIÓN}

La aplicación de la posición MILS tiene como objetivo evitar exacerbaciones de trauma cervical agudo. Sin embargo, su utilización mantiene la cabeza en posición neutra donde no hay adecuada alineación de los ejes laríngeo, faríngeo y oral, por lo tanto al hacer la laringoscopía hay una mala visualización glótica. Esto es un factor de riesgo de presentar intubación difícil y por lo tanto, hay una alta incidencia de intubaciones fallidas (una en 50-100 casos) y situaciones de «no se puede intubar ni ventilar» (una en 200 casos) en el departamento de urgencias ${ }^{(5,6)}$. La conversión de muchos casos a un posible caso de intubación difícil mediante el uso del MILS aumenta el riesgo de eventos hipóxicos con posibilidad de arritmias, trauma de vía aérea, paro cardiovascular, encefalopatía hipóxica y muerte.

Éste es el primer estudio hecho en población mexicana que describe el aumento del grado de CL bajo la maniobra de MILS, aunque ya ha sido documentada en otras poblaciones. Fue descrita por primera vez por Nolan y colaboradores ${ }^{(7)}$ al comparar la visualización con hoja Macintosh en posición óptima (olfateo) y posición neutra con MILS a 157 pacientes. Sus resultados indicaron que el grado glótico se mantuvo igual en $86 \%$ de los pacientes (54.8\%), disminuyó un grado en 56 pacientes (35.6\%) y dos grados en 15 pacientes (9.5\%). Posteriormente, Hastings y su equipo ${ }^{(8)}$ también demostraron cambios en la calidad de visión de laringoscopía directa al modificarse a MILS. El profesional de salud encargado del manejo de la vía aérea en pacientes politraumatizados debe considerar el riesgo-beneficio de la aplicación de la posición MILS y las desventajas que ésta puede tener en el intento de la intubación endotraqueal.

Adesida y colaboradores ${ }^{(9)}$ realizaron un ensayo clínico aleatorizado comparando posición de olfateo convencional con MILS, donde el grupo con MILS presentó mayores vistas grado III CL (35 versus $4.9 \%$ ) y mayor incidencia en intubaciones fallidas (47.4 versus 4.7\%). Otro ensayo clínico $^{(10)}$ que comparó posiciones de MILS versus olfateo demostró mayor proporción de pacientes a los que no se pudo intubar en los primeros 30 segundos de intento, mayor tiempo de intubación al primer intento y peor vista glótica con posición MILS; además, los 47 pacientes que no se pudieron intubar al primer intento en posición MILS se intubaron exitosamente al segundo intento al remover la posición MILS.

Después de los resultados obtenidos consideramos que la laringoscopía directa no debe plantearse como la primera opción en pacientes politraumatizados por el aumento de incidencia de laringoscopías difíciles e intubaciones fallidas.

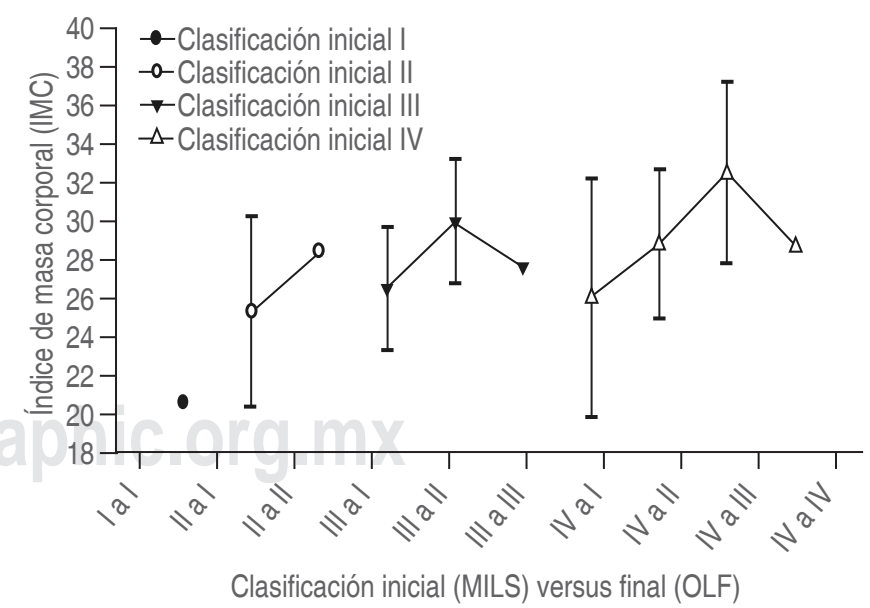

Figura 1: Valores de índice de masa corporal de las diferentes combinaciones de mejoría al cambiar de posición MILS a olfateo. MILS = Manual In-Line Stabilisation, OLF = olfateo. 
Si las condiciones clínicas lo permiten, deben buscarse otras alternativas para llevarse a cabo la intubación endotraqueal, dependiendo de los recursos disponibles en el centro hospitalario. Los videolaringoscopios son una excelente opción, ya que han demostrado menor movimiento de la columna cervical así como mejor visualización glótica y éxito de intubación endotraqueal en comparación con la laringoscopía directa ${ }^{(11)}$. Las mascarillas laríngeas de intubación pueden ser útiles en casos de difícil ventilación del paciente politraumatizado ${ }^{(12)}$, se ha demostrado la seguridad de su colocación en pacientes con trauma cervical. En un ensayo clínico aleatorizado la ML causó 62\% menos movimiento cráneo-cervical de occipucio a C5 en comparación con el videolaringoscopio ${ }^{(13)}$. El broncofibroscopio también puede ser de utilidad en esta población de pacientes en algunas situaciones específicas.

El efecto del IMC en el manejo de la vía aérea es un tema controvertido y actualmente no existe consenso de su impacto en el aumento de dificultad de laringoscopía e intubación. En un reciente metaanálisis, un análisis de subgrupo no demostró asociación alta entre obesidad con laringoscopía difícil e intubación difícil ${ }^{(14)}$. En nuestro estudio, al analizar el grado de mejoría al cambiar de posición MILS a olfateo se observó una clara tendencia en la que disminuía el valor de mejoría de CL conforme aumentaba el IMC (Figura 1). El análisis estadístico no fue significativo; sin embargo, probablemente se debió a un número reducido de pacientes.

Las limitaciones que presenta nuestro estudio es que la muestra poblacional fue pequeña para alcanzar significancia estadística para algunas variables analizadas, además de que el estudio se llevó a cabo en pacientes en quienes se excluyeron otros predictores de vía aérea difícil. Nuestro estudio fue una simulación de MILS, pero en un entorno electivo los resultados en los casos de intubación emergente en paciente politraumatizado podrían ser diferentes.

\section{CONCLUSIÓN}

La mayoría de los pacientes presenta una mejoría del CL cuando se cambia de posición MILS a olfateo, por ende la posición MILS aumenta la incidencia de laringoscopía difícil. Se observa una tendencia en la que se correlaciona un aumento de IMC con una disminución de la mejoría al cambiar de posición MILS a la posición de olfateo; sin embargo, esta tendencia no fue significativa.

\section{REFERENCIAS}

1. WHO. Injuries and Violence: The Factos. Geneva, Switzerland: World Health Organization; 2014.

2. Crosby E, Lui A. The adult cervical spine: Implications for airway management. Can J Anaesth. 1990;37:77-93.

3. Trauma ACoSCo. Advance Trauma Life Support Manual Chicago: American College of Surgeons; 1984.

4. Smith CE, Pinchak AB, Sidhu TS, Radesic BP, Pinchak AC, Hagen JF. Evaluation of tracheal intubation difficulty in patients with cervical spine immobilization. Anesthesiology. 1999;91:1253-1259.

5. Bair AE, Filbin MR, Kulkarni RG, Walls RM. The failed intubation attempt in the emergency department: analysis of prevalence, rescue techniques and personnel. J Emerg Med. 2002;23:131-140.

6. Cook TM, Macdougall-Davis SR. Complications and failure of airway management. Br J Anesth. 2012;109:68-85.

7. Nolan J, Wilson M, Park C. Orotracheal intubation in patients with potential cervical spine injuries. Anaesthesia. 1993;48:630-633.

8. Hastings RH, Wood PR. Head extension and laryngeal view during laryngoscopy with cervical spine stabilization maneuvers. Anesthesiology. 1994;80:825-831.

9. Adesida A, Desalu I, Adeyemo W, Kushimo O. Manual in-line stabilization of the cervical spine increases the rate of difficult orotracheal intubation in adults-a randomized controlled trial. The Annals of African Surgery. 2014;11:10-14.
10. Thiboutot F, Nicole PC, Trepanier CA, Turgeon AF, Lessard MR. Effect of manual in-line stabilization of the cervical spine ina dults on the rate of difficult orotracheal intubation by direct laryngoscopy: a randomized controlled trial. Can J Anesth. 2009;56:412-418.

11. Romito JW, Riccio CA, Bagley CA, Minhajuddin A, Barden $\mathrm{CB}$, Michael MM, et al. Cervical spine movement in a cadaveric model of severe spinal instability: a study comparing tracheal intubation with 4 different laryngoscopes. J Neurosurg Anesthesiol. 2020;32:57-62.

12. Lopez-Jimenez FA, Morales-Cuevas JM, Alvarado-Ramos JG, Mondragon-Villanueva ME. Intubación traqueal con mascarilla laríngea C-trach en pacientes con vía aérea y pacientes con lesión cervical. Rev Mex Anest. 2007;30:26-31.

13. Swain A, Bhagat H, Gupta V, Salunke P, Panda NB, Sahu S. Intubating laryngeal mask airway-assisted flexible bronchoscopic intubation is associated with reduced cervical spine motion when compared with C-MAC video laryngoscopy-guided intubation: a prospective randomized cross over trial. J Neurosurg Anesthesiol. 2019;32:242-248.

14. Wang T, Sun S, Huang S. The association of body mass index with dificult tracheal intubation management by direct laryngoscopy: a meta-analysis. BMC Anesthesiology. 2018;18:1-13. 


\begin{tabular}{cccc}
\multicolumn{4}{c}{ Anexo 1: $\chi^{2}$ (datos tabla 2). } \\
\hline Grado & F_MILS & F_OLF & \\
\hline I & 1.000 & 39.000 & Conteos \\
& 20.000 & 20.000 & Conteo esperado \\
& 2.500 & 97.500 & Fila\% \\
& 1.786 & 69.643 & Columna\% \\
II & 0.893 & 34.821 & Total\% \\
& 13.000 & 10.000 & Conteos \\
& 11.500 & 11.500 & Conteo esperado \\
& 56.522 & 43.478 & Fila\% \\
III & 23.214 & 17.857 & Columna $\%$ \\
& 11.607 & 8.929 & Total\% \\
& 26.000 & 6.000 & Conteos \\
& 16.000 & 16.000 & Conteo esperado \\
& 81.250 & 18.750 & Fila\% \\
& 46.429 & 10.714 & Columna $\%$ \\
IV & 23.214 & 5.357 & Total\% \\
& 16.000 & 1.000 & Conteos \\
& 8.500 & 8.500 & Conteo esperado \\
& 94.118 & 5.882 & Fila\% \\
& 28.571 & 1.786 & Columna $\%$ \\
& 14.286 & 0.893 & Total\% \\
\hline
\end{tabular}

$\chi^{2}=62.227$ con tres grados de libertad $(p \leq 0.001)$.

MILS = Manual In-Line Stabilisation, OLF = olfateo .

Las proporciones de observaciones en diferentes columnas de la tabla de contingencia varían de una fila a otra. Las dos características que definen la tabla de contingencia están significativamente relacionadas $(p \leq 0.001)$. Poder de la prueba realizada con alfa $=0.050: 1.000$.

\begin{tabular}{|c|c|c|c|c|c|}
\hline Grupo & $\mathrm{N}$ & Faltante & & & $\begin{array}{l}\text { Error } \\
\text { Estándar } \\
\text { e la Media }\end{array}$ \\
\hline$|a|$ & 1 & 0 & \multicolumn{2}{|c|}{$20.576 \pm 0.000$} & 0.000 \\
\hline||$a \mid$ & 12 & 0 & \multicolumn{2}{|c|}{$25.292 \pm 4.935$} & 1.425 \\
\hline$\|$ a $\|$ & 1 & 0 & \multicolumn{2}{|c|}{$28.394 \pm 0.000$} & 0.000 \\
\hline |ll a | & 20 & 0 & \multicolumn{2}{|c|}{$26.507 \pm 3.168$} & 0.708 \\
\hline III a II & 5 & 0 & \multicolumn{2}{|c|}{$29.960 \pm 3.224$} & 1.442 \\
\hline III a III & 1 & 0 & \multicolumn{2}{|c|}{$27.640 \pm 0.000$} & 0.000 \\
\hline$|\mathrm{V} a|$ & 6 & 0 & \multicolumn{2}{|c|}{$25.994 \pm 6.212$} & 2.536 \\
\hline IV a II & 4 & 0 & \multicolumn{2}{|c|}{$28.812 \pm 3.854$} & 1.927 \\
\hline IV a III & 5 & 0 & \multicolumn{2}{|c|}{$32.484 \pm 4.699$} & 2.101 \\
\hline IV a IV & 1 & 0 & \multicolumn{2}{|c|}{$28.685 \pm 0.000$} & 0.000 \\
\hline $\begin{array}{l}\text { Origen de } \\
\text { variación }\end{array}$ & $\begin{array}{l}\text { Grados } \\
\text { de } \\
\text { Libertad }\end{array}$ & $\begin{array}{l}\text { Suma de } \\
\text { cuadros }\end{array}$ & $\begin{array}{c}\text { Medias } \\
\text { cuadráticas }\end{array}$ & $\begin{array}{l}\text { Estadístico } \\
\text { de prueba }\end{array}$ & $p$ \\
\hline Entre grupos & 9 & 297.834 & 33.093 & 1.843 & 0.086 \\
\hline Residual & 46 & 826.096 & 17.959 & & \\
\hline Total & 55 & $1,123.931$ & & & \\
\hline
\end{tabular}

SEM = Error estándar de la media; DF = Grados de Libertad; SS = Suma de cuadros; MS = Medias cuadráticas; $\mathrm{F}$ = Estadístico de prueba.

Todos estos elementos en su conjunto hacen el análisis de la varianza.

Las diferencias en los valores medios entre los grupos de tratamiento no son lo suficientemente grandes para excluir la posibilidad de que la diferencia se deba a la variabilidad del muestreo aleatorio; no hay una diferencia estadísticamente significativa $(p=0.086)$.

Poder de la prueba realizada con alfa $=0.050: 0.357$

La potencia de la prueba realizada $(0.357)$ está por debajo de la potencia deseada de 0.800 .

Una potencia inferior a la deseada indica que es menos probable que detecte una diferencia cuando realmente existe. Los resultados negativos deben interpretarse con cautela.

\begin{tabular}{lccc} 
& \multicolumn{3}{c}{ Anexo 2: $\chi^{2}}$. \\
Grado & MILS & OLF & \\
\hline I-II & 14.000 & 49.000 & Conteos \\
& 31.500 & 31.500 & Conteo esperado \\
& 22.222 & 77.778 & Fila\% \\
& 25.000 & 87.500 & Columna\% \\
III-IV & 12.500 & 43.750 & Total\% \\
& 42.000 & 7.000 & Conteos \\
& 24.500 & 24.500 & Conteo esperado \\
& 85.714 & 14.286 & Fila\% \\
& 75.000 & 12.500 & Columna\% \\
& 37.500 & 6.250 & Total\% \\
\hline
\end{tabular}

MILS = Manual In-Line Stabilisation, OLF = Olfateo.

Se utilizó la corrección de Yates para la continuidad en el cálculo de esta prueba. $\chi^{2}=41.941$ con un grado de libertad $(p \leq 0.001)$.

Las proporciones de observaciones en diferentes columnas de la tabla de contingencia varían de una fila a otra. Las dos características que definen la tabla de contingencia están significativamente relacionadas $(p \leq 0.001)$.

Poder de la prueba realizada con alfa $=0.050: 1.000$. 